\title{
CONTROL OF ROBOT-CAMERA SYSTEM WITH ACTUATOR'S DYNAMICS TO TRACK MOVING OBJECT
}

\author{
NGUYEN TIEN KIEM ${ }^{1}$ AND PHAM THUONG CAT ${ }^{2}$ \\ ${ }^{1}$ Hanoi University of Industry (HaUI); kiemnguyentien@gmail.com \\ ${ }^{2}$ Institute of Information Technology, Vietnam Academy of Science and Technology; \\ ptcat@ioit.ac.vn
}

\begin{abstract}
chosshe
Abstract. This study presents a solution to the control of robot-camera system with actuator's dynamics to track a moving object where many uncertain parameters exist in the system's dynamics.

After modeling and analyzing the system, this paper suggests a new control method using an online learning neural network in closed-loop to control the Pan-Tilt platform that moves the Camera to keep tracking an unknown moving object. The control structure based on the image feature error determines the necessary rotational velocities on the Pan joint and Tilt joint and computes the voltage controlling the DC motor in joints such that the object image should always be at the center point in the image plane. The global asymptotic stability of the closed-loop is proven by the Lyapunov direct stability theory. Simulation results on Matlab show the system tracking fast and stable.
\end{abstract}

Keywords. Robot control, artificial neural network, visual servoing.

Abbreviations. RBF: Radial Basic Function; DC: Direct Current

Symbols:

$\xi \quad$ Image-specializing vector;

$\mathbf{J}_{f} \quad$ Image Jacobian matrix;

$\dot{\mathbf{q}}_{\mathbf{d}} \quad$ Desired Pan Tilt joint-angle velocity vector;

$\dot{\mathbf{q}} \quad$ The actual joint-angle velocity vector of Pan-Tilt platform;

$\mathbf{J}_{r} \quad$ Jacobian matrix of Pan-Tilt platform;

$\boldsymbol{\tau} \quad$ Joint torque vector of Pan-Tilt platform;

${ }^{\mathbf{w}} \mathbf{r}_{\mathbf{o}}$ Target coordinates in the coordinate system of the Pan-Tilt platform;

${ }^{\mathbf{c}} \mathbf{r}_{\mathbf{o}} \quad$ Target coordinates in the camera coordinate system;

$\mathbf{u}_{E} \quad$ Voltage vector controlling a direct current motor armature;

i Current vector of direct current motor armature; and

R Resistor matrix of direct current motor armature.

\section{INTRODUCTION}

Pan-Tilt platform with two layers freely rotates in two azimuthal direction (Pan) and angle (Tilt). This structure mainly is used as rotating radar platform (fixed or mobile located on car, train) or rotating tray of optical devices to monitor and test space. The majority of studies on this system ignores the impact of the actuators due to the fact that the system is often installed with gear reducer large reduction ratio. However, when the target moves fast and it is necessary to use the direct transmission, the impact of the dynamics of the actuators can not be ignored. At this moment it is necessary to install an additional model of the engines mounting in the joints of the pad into the 
dynamics of the whole system; it also needs to consider the interaction between the robot and the dynamics of actuators.

Currently in the world, there have been many studies and scientific reports on a method for controlling how to track moving targets using Pan - Tilt platform and camera. A control method using neural networks to compensate for the uncertainty components of the robot arms tracking target is described in [1]. The method for controlling how to track targets in 3D space by Pan Tilt camera system and using continuous pixel-tracking method and pixel filter is presented in [2]. Paper [3] has introduced the control method to track the target using signals via video obtained from flying objects. Paper [4] has used remote controlled robot arm having 6 degree-of-freedoms combined with a flexible control method to follow the target in 3D space. Paper [5] has introduced a new control approach using nonlinear observers for robot arm and a camera to track a moving target in 3D space. Stable control method combined with a neural network, identifying the parameters of the robot is described in [6]. The above reports often overlook the impact of actuators' dynamics mounted in the robot joints.

In this paper, the authors study methods for controlling Pan/Tilt pedestal mounted with the camera tracking a moving target with a number of uncertain parameters and also take into account the model of a direct current motor mounted in the joints of the pedestal.

The report is divided into four sections. After the introduction, the next part is building a mathematical model of the system and determining the visual servo problem. Following part is a proposal of Pan-tilt-camera control algorithm and notice of actuator's effect. Part 4 presents verification, simulation and finally there are some evaluation and conclusions.

\section{MATHEMATICAL MODEL OF ROBOT CAMERA SYSTEM AND PROBLEM FORMULATION}

The model of the system consists of model of imaging camera, nonlinear dynamics of Pan/Tilt pedestal and the transfer function of DC motors mounted in the joints.

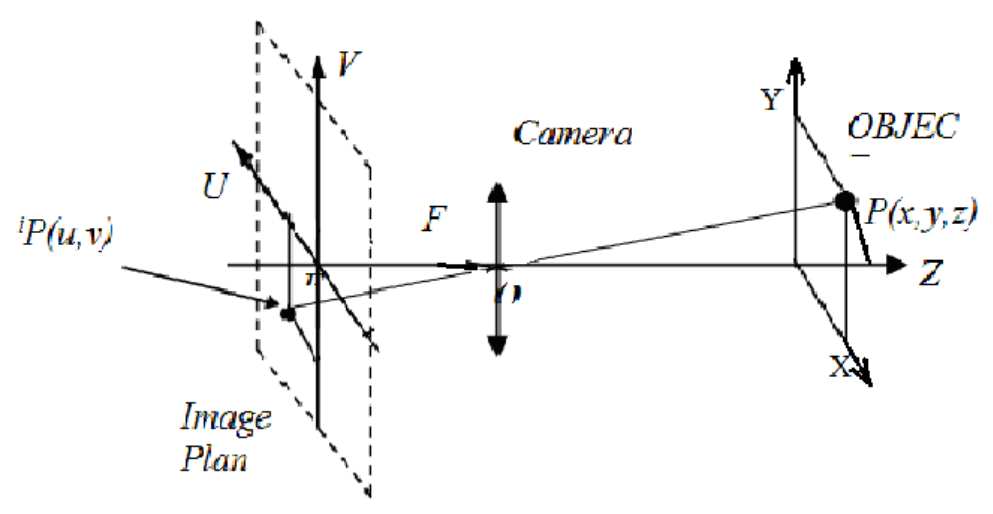

Figure 1: Camera imaging model 


\section{Description of camera imaging equation}

The image of a point $P(x, y, z)$ in space is mapped into the image plane obtained point ${ }^{I} P(u, v)$ with coordinates as follows (Figure 1):

$$
u=\frac{f x}{z} ; \quad v=\frac{f y}{z},
$$

in which $f$ is the focal length of the camera. The image of target after being digitized and determined image features will give the central coordinates of the target on the image plane. This coordinate is written as $\boldsymbol{\xi}=[u, v]^{T}$ and will be used as a measuring parameter of Pan-Tilt camera system.

Control mission is done via the difference function between desired image features $\boldsymbol{\xi}_{d}$ and obtained image features. This bias function can be defined as follows:

$$
\mathbf{e}=\left(\boldsymbol{\xi}-\boldsymbol{\xi}_{d}\right) .
$$

\section{Description of the kinematic and dynamic equations of Pan-Tilt robots [7]:}

A block diagram of the overall system is described in Figure 2.

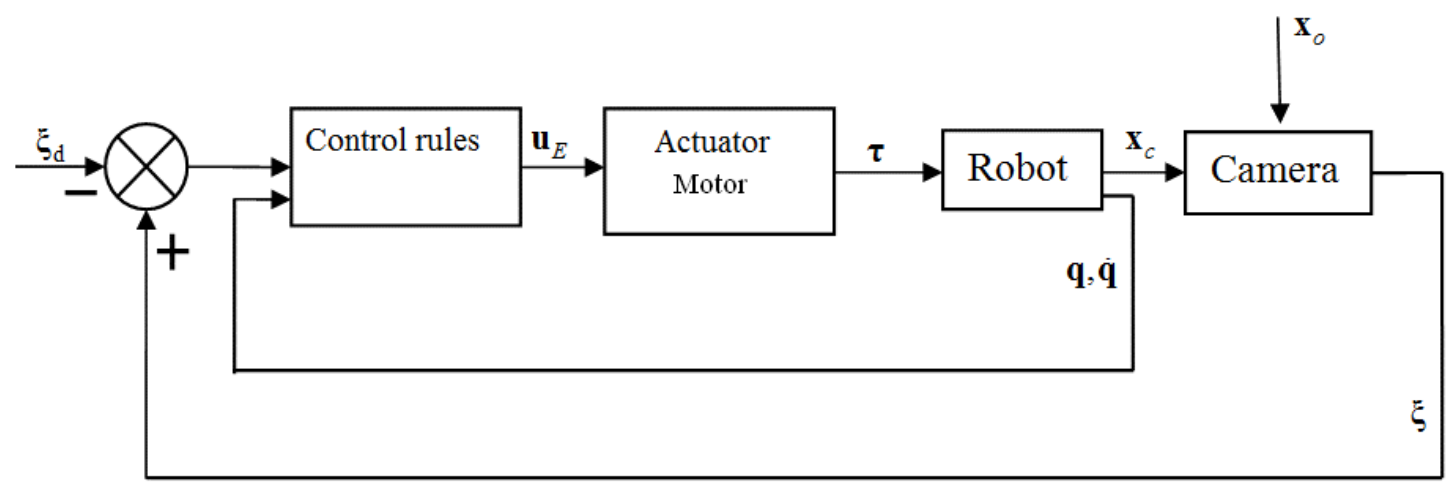

Figure 2: Block diagram of Pan-Tilt-camera system

$\mathbf{x}_{c}$ and $\mathbf{x}_{o}$ are camera coordinate and target coordinate respectively in the Cartesian coordinate system attached to the robot platform. Kinematic equation of the robot is described as follows:

$$
\mathbf{x}_{c}=\mathbf{p}(\mathbf{q})
$$

Time derivation of (3) is:

$$
\dot{\mathbf{x}}_{c}=\frac{\partial \mathbf{p}}{\partial \mathbf{q}} \frac{d q}{d t}=\mathbf{J}_{r} \dot{\mathbf{q}}
$$

where $\dot{\mathbf{x}}_{c}$ is the translational velocity and the angular velocity of the camera, $\mathbf{J}_{r}$ the Jacobian matrix of the robot.

Dynamic equations of the robot (4) and of the DC motor (5), (6) are described as follows:

$$
\begin{gathered}
\boldsymbol{\tau}=\mathbf{H}(\mathbf{q}) \ddot{\mathbf{q}}+\mathbf{h}(\mathbf{q}, \dot{\mathbf{q}}) \\
\mathbf{L} \dot{\mathbf{i}}+\mathbf{R i}+\mathbf{K} \dot{\mathbf{q}}+\mathbf{t}_{E}=\mathbf{u}_{E}
\end{gathered}
$$




$$
\tau=\mathbf{K}_{T} \mathbf{i}
$$

in which $\mathbf{q}=\left[q_{1}, q_{2}\right]^{T}$ is joint coordinate vector, $\boldsymbol{\tau}=\left[\tau_{1}, \tau_{2}\right]^{T}$ joint torque vector of robot, $\mathbf{H}(\mathbf{q})$ positive definite symmetric matrix, showing the robot inertia matrix, and $\mathbf{h}(\mathbf{q}, \dot{\mathbf{q}})$ a vector which presents Coriolis torque components, centrifugal and torque components caused by the gravitational force. $\mathbf{u}_{E}=\left[u_{E 1}, u_{E 2}\right]^{T}$ is the voltage of controlling armature of two DC motors, $\mathbf{i}=\left[i_{1}, i_{2}\right]^{T}$ current vector of the armature of the DC motors, and $\mathbf{L}=<L_{1}, L_{2}>$ constant diagonal matrix determining the inductance of the armature winding. $\mathbf{R}=<R_{1}, R_{2}>$ presents resistor matrix of the armature of a DC motor. $\mathbf{K}_{T}=<K_{T 1}, K_{T 2}>$ diagonal matrix defining positive torque coefficient of two motors, $\mathbf{K}=<K_{T}, K_{T}>$ diagonal matrix defining positive factors of feedback voltage of two motors, and $\mathbf{t}_{E}$ a vector which shows uncertainty components of the motor. The uncertainty components are proposed to be blocked $\left\|\mathbf{t}_{E}\right\| \leq T_{0}, T_{0}$ are known components.

Image feature vector depends on locations and directions of the camera, therefore it depends on q. If $m$ is the number of image features, the image feature vector will have $2 m$ elements. The relation between image feature vector $\boldsymbol{\xi}$ and $\mathbf{x}_{c}$ is defined as follows:

$$
\frac{d \boldsymbol{\xi}}{d t}=\mathbf{J}_{f} \frac{d \mathbf{x}_{c}}{d t}
$$

In which, $\mathbf{J}_{f}$ is an image Jacobian matrix. On the other hand, $\dot{\mathbf{x}}_{c}=\mathbf{J}_{r} \dot{\mathbf{q}}$ therefore, it yields:

$$
\frac{d \boldsymbol{\xi}}{d t}=\mathbf{J}_{f} \frac{d \mathbf{x}_{c}}{d t}=\mathbf{J}_{f} \mathbf{J}_{r} \dot{\mathbf{q}}
$$

Definition of general Jacobi matrix:

$$
\mathbf{J}(\xi, \mathbf{q})=\mathbf{J}_{f} \mathbf{J}_{r}
$$

Equation (8) can be rewritten as follows:

$$
\dot{\xi}=\mathbf{J}(\xi, \mathbf{q}) \dot{\mathbf{q}}
$$

It is proposed that uncertain parameters of robot are described as follows:

$$
\begin{gathered}
\mathbf{H}(\mathbf{q})=\widehat{\mathbf{H}}(\mathbf{q})+\Delta \mathbf{H}(\mathbf{q}) \\
\mathbf{h}(\mathbf{q})=\widehat{\mathbf{h}}(\mathbf{q})+\Delta \mathbf{h}(\mathbf{q}) \\
\mathbf{J}(\xi, \mathbf{q})=\widehat{\mathbf{J}}(\xi, \mathbf{q})+\Delta \mathbf{J}(\boldsymbol{\xi}, \mathbf{q})
\end{gathered}
$$

in which $\widehat{\mathbf{H}}(\mathbf{q}), \widehat{\mathbf{h}}(\mathbf{q}), \widehat{\mathbf{J}}(\xi, \mathbf{q})$ are known components; $\Delta \mathbf{H}(\mathbf{q}), \boldsymbol{\Delta} \mathbf{h}(\mathbf{q}), \Delta \mathbf{J}(\boldsymbol{\xi}, \mathbf{q})$ are unknown ones. Replacing (11), (12), and (13) on the place of the equation (4) and (10) results in a dynamic system of Pan-Tilt-camera platform with a lot of uncertain parameters:

$$
\begin{gathered}
\boldsymbol{\tau}=\widehat{\mathbf{H}}(\mathbf{q}) \ddot{\mathbf{q}}+\widehat{\mathbf{h}}(\mathbf{q}, \dot{\mathbf{q}})+\Delta \mathbf{H}(\mathbf{q}) \ddot{\mathbf{q}}+\Delta \mathbf{h}(\mathbf{q}, \dot{\mathbf{q}}) \\
\dot{\xi}=\hat{\mathbf{J}} \dot{\mathbf{q}}+\Delta \mathbf{J} \dot{\mathbf{q}}
\end{gathered}
$$




\section{Formulation of visual servoing problem:}

The problem of tracking moving target consists in finding out voltage $\mathbf{u}_{E}=\left[u_{E 1}, u_{E 2}\right]^{T}$ so that the spindle motors of the camera platform follow moving targets and the error of image feature $\mathbf{e}=\left(\boldsymbol{\xi}-\boldsymbol{\xi}_{d}\right)$ disappears.

From (14), it can be deduced that:

$$
\ddot{\mathbf{q}}=\widehat{\mathbf{H}}^{-1}(\mathbf{q}) \boldsymbol{\tau}-\widehat{\mathbf{H}}^{-1} \widehat{\mathbf{h}}(\mathbf{q}, \dot{\mathbf{q}})-\widehat{\mathbf{H}}^{-1}[\Delta \mathbf{H}(\mathbf{q}) \ddot{\mathbf{q}}+\Delta \mathbf{h}(\mathbf{q}, \dot{\mathbf{q}})]
$$

Definition of variable describing deviations of image features: $\mathbf{z}=\mathbf{G}\left(\xi-\xi_{d}\right)$, in which, $\mathbf{G}$ is a constant matrix $[n \times 2 m]$, with rank $n$. Thus, if $\mathbf{z} \rightarrow \mathbf{0}$ yields that $\mathbf{e} \rightarrow \mathbf{0}$. Taking the first and the second derivative of $\mathbf{z}$ over time results in:

$$
\begin{gathered}
\dot{\mathbf{z}}=\mathbf{G} \dot{\xi}=\mathbf{G J} \dot{\mathbf{q}}=\mathbf{G} \widehat{\mathbf{J}} \dot{\mathbf{q}}+\mathbf{G} \Delta \mathbf{J} \dot{\mathbf{q}} \\
\ddot{\mathbf{z}}=\mathbf{G} \ddot{\xi}=\mathbf{G} \dot{\widehat{\mathbf{J}}} \dot{\mathbf{q}}+\mathbf{G} \widehat{\mathbf{J}} \ddot{\mathbf{q}}+\mathbf{G} \Delta \dot{\mathbf{J}} \dot{\mathbf{q}}+\mathbf{G} \Delta \mathbf{J} \ddot{\mathbf{q}}
\end{gathered}
$$

From (16), (18) it derives:

$$
\boldsymbol{\tau}=\widehat{\mathbf{H}}(\mathbf{G} \widehat{\mathbf{J}})^{-1} \ddot{\mathbf{z}}-\widehat{\mathbf{H}}(\mathbf{G} \widehat{\mathbf{J}})^{-1} \mathbf{G} \dot{\mathbf{J}} \dot{\mathbf{q}}+\widehat{\mathbf{h}}+\mathbf{f}
$$

with $\mathbf{f}=(\Delta \mathbf{H}+\mathbf{G} \Delta \mathbf{J}) \ddot{\mathbf{q}}-\hat{\mathbf{H}}(\mathbf{G} \hat{\mathbf{J}})^{-1} \mathbf{G} \Delta \dot{\mathbf{J}} \dot{\mathbf{q}}+\Delta \mathbf{h}$.

Moreover, from (5), (6) and (19) there are:

$$
\begin{aligned}
& \mathbf{K}_{T} \mathbf{R}^{-1} \mathbf{u}_{E}=\hat{\mathbf{H}}(\mathbf{G} \hat{\mathbf{J}})^{-1} \ddot{\mathbf{z}}-\hat{\mathbf{H}}(\mathbf{G} \hat{\mathbf{J}})^{-1} \mathbf{G} \dot{\hat{\mathbf{J}}} \dot{\mathbf{q}}+\hat{\mathbf{h}}+\mathbf{K}_{T} \mathbf{R}^{-1} \mathbf{K} \dot{\mathbf{q}} \\
&+\mathbf{K}_{T} \mathbf{R}^{-1} \mathbf{L} \dot{\mathbf{i}}+(\Delta \mathbf{H}+\mathbf{G} \Delta \mathbf{J}) \ddot{\mathbf{q}}-\hat{\mathbf{H}}(\mathbf{G} \hat{\mathbf{J}})^{-1} \mathbf{G} \Delta \dot{\mathbf{J}} \dot{\mathbf{q}}+\Delta \mathbf{h}+\mathbf{R}^{-1} \mathbf{t}_{E}
\end{aligned}
$$

The variables are set as follows:

$$
\boldsymbol{\psi}=R K_{T}^{-1} \hat{\mathbf{H}}(\mathbf{G} \hat{\mathbf{J}})^{-1}
$$

With the inverse matrices, it can be seen clearly that the matrix $\boldsymbol{\psi}$ is an invertible matrix:

$$
\begin{gathered}
\boldsymbol{\psi}^{-1}=(\mathbf{G} \hat{\mathbf{J}}) \hat{\mathbf{H}}^{-1} \mathbf{K}_{T} \mathbf{R}^{-1} \\
\mathbf{f}_{1}=\mathbf{R} \mathbf{K}_{T}^{-1}\left[(\Delta \mathbf{H}+\mathbf{G} \Delta \mathbf{J}) \ddot{\mathbf{q}}-\hat{\mathbf{H}}(\mathbf{G} \hat{\mathbf{J}})^{-1} \mathbf{G} \Delta \dot{\mathbf{J}} \dot{\mathbf{q}}+\Delta \mathbf{h}\right]+ \\
+\mathbf{K}_{T}^{-1} \mathbf{t}_{E}+\mathbf{L} \dot{\mathbf{i}} \\
\gamma=\mathbf{R K}_{T}^{-1}\left[-\hat{\mathbf{H}}(\mathbf{G} \hat{\mathbf{J}})^{-\mathbf{1}} \mathbf{G} \dot{\hat{\mathbf{J}}} \dot{\mathbf{q}}+\hat{\mathbf{h}}\right]+\mathbf{K} \dot{\mathbf{q}}
\end{gathered}
$$

Combining equations (20), (21), (23), (24) results in:

$$
\boldsymbol{\psi} \ddot{\mathbf{z}}+\gamma+\mathbf{f}_{1}=\mathbf{u}_{E}
$$

Consequently, the tracking control problem becomes a state of finding voltage $\mathbf{u}_{E}$ to control the system (25) stably approaching $\lim _{t \rightarrow \infty} \mathbf{z}(t) \rightarrow \mathbf{0}$ while there are uncertainties about the component $\mathbf{f}_{1}$. Of course, when $\lim _{t \rightarrow \infty} \mathbf{z}(t) \rightarrow \mathbf{0}$ there is error in image feature $\mathbf{e}(t) \rightarrow \mathbf{0}$. 


\section{CONTROL ALGORITHM TRACKING THE MOVING TARGET USING NEURAL NETWORK}

Let us look for the control voltage $\mathbf{u}_{E}$ in the form of:

$$
\begin{gathered}
\mathbf{u}_{E}=\mathbf{u}_{0}+\mathbf{u}_{1} \\
\mathbf{u}_{0}=\boldsymbol{\psi}\left(-\mathbf{K}_{D} \dot{\mathbf{z}}-\mathbf{K}_{P} \mathbf{z}\right)+\gamma
\end{gathered}
$$

$\mathbf{u}_{1}$ is control signals to compensate for the effects of uncertain components will be defined later. Putting (26), (27) in the place of (25) yields:

$$
\ddot{\mathbf{z}}+\mathbf{K}_{D} \dot{\mathbf{z}}+\mathbf{K}_{P} \mathbf{z}=\boldsymbol{\psi}^{-1}\left(\mathbf{u}_{1}-\mathbf{f}_{1}\right)
$$

If uncertain components $\mathbf{f}_{1}=\mathbf{0}$, and choosing $\mathbf{u}_{1}=\mathbf{0}$, the difference equation will be:

$$
\ddot{\mathbf{z}}+\mathbf{K}_{D} \dot{\mathbf{z}}+\mathbf{K}_{P} \mathbf{z}=\mathbf{0}
$$

Equation (29) is asymptotically stable $(\mathbf{z} \rightarrow \mathbf{0})$ with fast speed and no overshoot if the matrix $\mathbf{K}_{D}$, $\mathbf{K}_{P}$ is chosen suitably. In any case, $\mathbf{f}_{1}\left(\mathbf{0}\right.$, then it needs to define $\mathbf{u}_{1}$ in order to do that (25) asymptotically stable. Define:

$$
\begin{aligned}
\mathbf{u}^{\prime} & =\boldsymbol{\psi}^{-1} \mathbf{u}_{1} \\
\mathbf{f}^{\prime} & =\boldsymbol{\psi}^{-1} \mathbf{f}_{1}
\end{aligned}
$$

Replacing (30), (31) on the place of (25) results in:

$$
\ddot{\mathbf{z}}+\mathbf{K}_{D} \dot{\mathbf{z}}+\mathbf{K}_{P} \mathbf{z}=\mathbf{u}^{\prime}-\mathbf{f}^{\prime}
$$

It is known that, a neural network RBF (Radial Basis Function) with a finite number of neurons can approximate unknown continuous and bounded function. Supposed that the robot is working in the nonsingular area so the $\mathbf{f}^{\prime}$ remain $\mathbf{s}$ continuous and bounded. It is to build a neural network with appropriate weight updating laws to approximate $\mathbf{f}^{\prime}$ and find out $\mathbf{u}^{\prime}$ that makes equation (32) asymptotically stable.

Artificial neural network, to approximate $\mathbf{f}^{\prime}$, is described (in Figure 3):

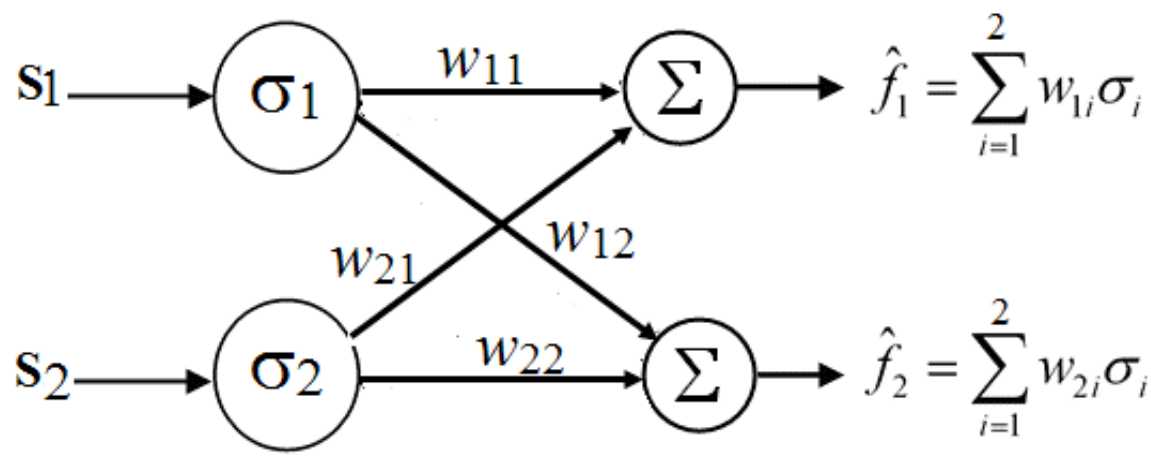

Figure 3: RBF network, approximately $\mathbf{f}^{\prime}$

In which, $\mathbf{s}=\left[s_{1}, s_{2}, \ldots\right]^{T}$ is defined as follows:

$$
\mathbf{s}=\dot{\mathbf{z}}+\mathbf{C z}
$$


$\mathbf{s}$ involve to $\mathbf{q}, \dot{\mathbf{q}}$ so $\mathbf{f}^{\prime}(\mathbf{q}, \dot{\mathbf{q}}) \mathbf{f}^{\prime}(\mathbf{s})$

$$
\begin{gathered}
\mathbf{f}^{\prime}=\mathbf{W} \sigma+\varepsilon=\hat{\mathbf{f}}+\varepsilon \\
\hat{\mathbf{f}}=\mathbf{W} \sigma
\end{gathered}
$$

With $\mathbf{W}$ is the weight matrix with law updated online and $\varepsilon$ is approximately bounded deviation $\|\varepsilon\| \leq \varepsilon_{0}$.

Here the input layer of the neural network is selected as $n=2$. The output layer has $n=2$ of linear neurons. The hidden layer consists of neurons with output function Gauss in the form of:

$$
\sigma_{j}=\exp \left(-\frac{\left(\varepsilon_{j}-c_{j}\right)^{2}}{\lambda_{j}^{2}}\right) ; j=1,2 .
$$

in which $c_{j}, \lambda_{j}$ parameters of the Gaussian distribution function can manually be selected.

Theorem 1. Pan-Tilt robotic camera system with 2 degree-of-freedoms with uncertain parameters (25) and neural networks (34), (35) will follow moving targets with errors $\mathbf{e} \rightarrow \mathbf{0}$ if we select control $\boldsymbol{u}$ and updating algorithm $\dot{\mathbf{W}}$ of neuron network as follows:

$$
\begin{gathered}
\mathbf{u}=\boldsymbol{\psi}\left(-\mathbf{K}_{D} \dot{\mathbf{z}}-\mathbf{K}_{P} \mathbf{z}\right)+\boldsymbol{\gamma}+\mathbf{u}_{1} \\
\mathbf{u}_{1}=\boldsymbol{\psi}\left[(1+\eta) \mathbf{W} \sigma-\delta \frac{\mathbf{s}}{\|\mathbf{s}\|}\right] \\
\dot{\mathbf{W}}=-\eta \mathbf{s} \sigma^{T}
\end{gathered}
$$

In which $\boldsymbol{K}_{D}=\boldsymbol{D}+\boldsymbol{C}, \boldsymbol{K}_{P}=\boldsymbol{D} \boldsymbol{C} ; \boldsymbol{D}$ is a positive definite symmetric matrix $\boldsymbol{D}=\boldsymbol{D}^{T}>\boldsymbol{O}$, and $\eta, \delta>0$.

Proof. Choose Lyapunov candidate function:

$$
V=\frac{1}{2}\left(\mathbf{s}^{T} \mathbf{s}+\sum_{i=1}^{n} \mathbf{w}_{i}^{T} \mathbf{w}_{i}\right)
$$

Take the derivative $\mathrm{V}$ over time:

$$
\dot{V}=\mathbf{s}^{T} \dot{\mathbf{s}}+\sum_{i=1}^{n} \mathbf{w}_{i}^{T} \dot{\mathbf{w}}_{i}
$$

From (33), (34) and $\mathbf{K}_{D}=\mathbf{D}+\mathbf{C}, \mathbf{K}_{P}=\mathbf{D C}$, it derives:

$$
\dot{\mathbf{s}}=\mathbf{u}^{\prime}-\mathbf{f}^{\prime}-\mathbf{D s}
$$

Replacing (42) with (41), yields:

$$
\dot{V}=-\mathbf{s}^{T} \mathbf{D} \mathbf{s}+\mathbf{s}^{T}\left(\mathbf{u}^{\prime}-\mathbf{f}^{\prime}\right)+\sum_{i=1}^{n} \mathbf{w}_{i}^{T} \dot{\mathbf{w}}_{i}
$$

With online updating law:

$$
\dot{\mathbf{w}}_{i}=-\eta \mathbf{s} \sigma_{i} \quad ; i=1,2 .
$$


in which $\mathbf{w}_{i}$ is $i$ column of matrix $\mathbf{W}$, it results in:

$$
\begin{aligned}
\sum_{i=1}^{2} \mathbf{w}_{i}^{T} \dot{\mathbf{w}}_{i} & =\sum_{i=1}^{2}-\eta \mathbf{w}_{i}^{T} \mathbf{s} \sigma_{i} \\
& =-\eta \mathbf{s}^{T} \sum_{i=1}^{2} \mathbf{w}_{i} \sigma_{i} \\
& =-\eta \mathbf{s}^{T} \mathbf{W} \sigma
\end{aligned}
$$

and

$$
\dot{V}=-\mathbf{s}^{\mathbf{T}} \mathbf{D} \mathbf{s}+\mathbf{s}^{\mathbf{T}}\left(\mathbf{u}^{\prime}-(1+\eta) \mathbf{W} \sigma-\varepsilon\right)
$$

Choose

$$
\mathbf{u}^{\prime}=(1+\eta) \mathbf{W} \sigma-\delta \frac{\mathbf{s}}{\|\mathbf{s}\|} ; \delta>0
$$

And replace (47) with (46):

$$
\begin{aligned}
\dot{V} & =-\mathbf{s}^{\mathbf{T}} \mathbf{D} \mathbf{s}+\mathbf{s}^{\mathbf{T}}\left(-\delta \frac{\mathbf{s}}{\|\mathbf{s}\|}-\varepsilon\right) \\
& \leq-\mathbf{s}^{\mathbf{T}} \mathbf{D} \mathbf{s}-\delta\|\mathbf{s}\|+\|\varepsilon\| \cdot\|\mathbf{s}\| \\
& \leq-\mathbf{s}^{\mathbf{T}} \mathbf{D} \mathbf{s}-\delta\|\mathbf{s}\|+\varepsilon_{0} \cdot\|\mathbf{s}\|
\end{aligned}
$$

Choosing $\delta=\varepsilon_{0}+\mu ; \mu>0$ yields:

$$
\dot{V} \leq-\mathbf{s}^{\mathbf{T}} \mathbf{D s}-\mu \cdot\|\mathbf{s}\| \leq 0
$$

From (49) $\dot{V}$ is a negative - semi definite function. This implies that $V(t) \leq V(0)$, and therefore, that $\mathbf{s}$ and $\mathbf{W}$ are bounded. Because the system is non- autonomous, it cannot conclude that $\mathbf{s}$ converge to zero. The Barbalat's lemma is used to prove the asymptotic stability of the system. Let us check the uniform continuity of $\dot{V}$. The derivative of $\dot{V}$ overtime from (48) is:

$$
\begin{aligned}
\ddot{V} & =-2 \mathbf{s}^{T} D \dot{\mathbf{s}}-\delta \frac{d}{d t}\left(\frac{\mathbf{s}^{T} \mathbf{s}}{\|\mathbf{s}\|}\right)-\frac{d}{d t} \mathbf{s}^{T} \boldsymbol{\varepsilon} \\
& =-2 s^{T} D \dot{\mathbf{s}}-2 \delta \frac{s^{T} \dot{\mathbf{s}}}{\|\mathbf{s}\|}-2 s^{T} \dot{\boldsymbol{\varepsilon}}
\end{aligned}
$$

It is founded that $\ddot{V}$ is bounded because $\mathbf{s}, \varepsilon$ are bounded, $\frac{\mathbf{s}^{T}}{\|\mathbf{s}\|}$ is the unite row vector of s and always bounded, D is the positive-definite constant matrix and constant. Thus $\dot{V}$ is uniformly continuous. According to Barbalat's lemma, the system is asymptotically stable meaning that $\mathbf{s}, \mathbf{z} \rightarrow \mathbf{0}$ and.

\section{THE SIMULATION RESULT AND ANALYSIS}

To demonstrate the proposed algorithms, the following simulation is performed on Matlab-Simulink:

- The chosen target coordinates are $(0.8,-0.3,0.2) \mathrm{m}$, the focal length of the camera is $f=5$ $\mathrm{mm}$ and the initial position of the joint angles are $\left(q_{1}=0 ; q_{2}=0\right)$. 
- The dynamic parameters of Pan-Tilt pedestal [14]:

$$
\begin{aligned}
& \mathbf{H}=\left[\begin{array}{ll}
\left(J_{1}+J_{2} \sin ^{2} q_{2}+J_{1} \cos ^{2} q_{2}\right) & 0 \\
0 & J_{1}
\end{array}\right] \quad\left[s^{2} \mathrm{~N}\right], \\
& \kappa_{1}=\left(J_{1}-J_{2}\right) \sin q_{2} \cos q_{2}, \\
& \mathbf{h}=\left[\begin{array}{ll}
\kappa_{1} \dot{q}_{2}+C_{1} & \kappa_{1} \dot{q}_{1} \\
\kappa_{1} \dot{q}_{1} & C_{2}
\end{array}\right]\left[\begin{array}{l}
\dot{q}_{1} \\
\dot{q}_{2}
\end{array}\right] \quad[\mathrm{Nm}]
\end{aligned}
$$

$J_{1}, J_{2}$ : Inertial moment of joint 1 and joint 2 with:

$J_{1}=0.02\left[\mathrm{kgm}^{2}\right], J_{2}=0.01\left[\mathrm{kgm}^{2}\right]$

$C_{1}, C_{2}$ : Viscous friction coefficient with $C_{1}=C_{2}=0.001$.

- Parameters of the two DC motors:

$$
\begin{aligned}
& \mathbf{K}_{T}=<2,2>[\mathrm{Nm} / \mathrm{A}] \\
& \mathbf{R}=<0.5,0.25>[\Omega] \\
& \mathbf{L}<1,0.5>[\mathrm{H}] \\
& \mathbf{t}_{E}=[0.20 .1]^{T}[\mathrm{~V}]
\end{aligned}
$$

- Uncertainty: $10 \%$ of actual value.

- Neural Network parameters RBF:

$$
\begin{aligned}
& \eta=10 ; \\
& \lambda_{1}=\lambda_{2}=5.0 ; \\
& c_{1}=c_{2}=0.001 ;
\end{aligned}
$$

- Parameters of the control law:

$$
\begin{aligned}
& \delta=0.25 \\
& \mathbf{D}=<10,10> \\
& \mathbf{C}=<8,8> \\
& \mathbf{K}_{P}=<80,80>\left[1 / \mathrm{s}^{2}\right] \\
& \mathbf{K}_{D}=<18,18>[1 / \mathrm{s}] .
\end{aligned}
$$

The aim of the visual servoing system is to control Pan/Tilt camera pedestal so that the image of the target is located in the center of the image plane $(u=0 ; v=0)$. Matlab simulation results are described in Figures 4, 5, 6, and 7 as follows:

The simulation results indicate that the robot-camera system is stable, although the system is affected by the dynamics and noise of motors. Despite the fact that there have been still a number of uncertainty parameters in the dynamic system of the Pan / Tilt pedestal the tracking error converges zero. It is seen in Figures 5 and 6 after 4.5 seconds, the system has basically tracked the target. The target image feature also nicely converges to the origin of the image plane in Figure 4. 


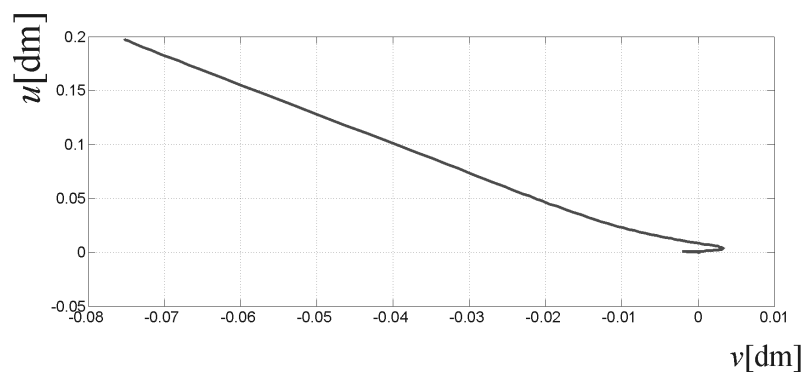

Figure 4: Graph of image features

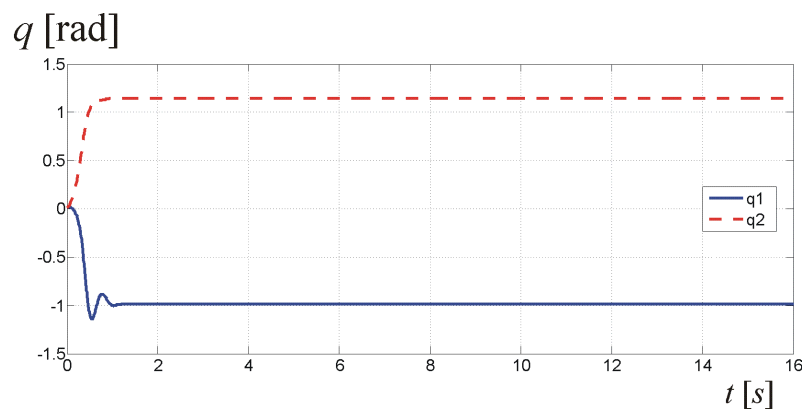

Figure 5: Graph of joint coordinates

$$
\dot{q}[\mathrm{rad} / \mathrm{s}]
$$

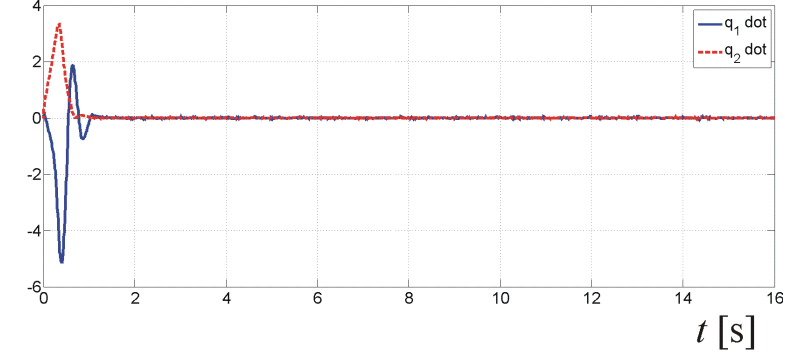

Figure 6: Graph of Pan Tilt joint velocity

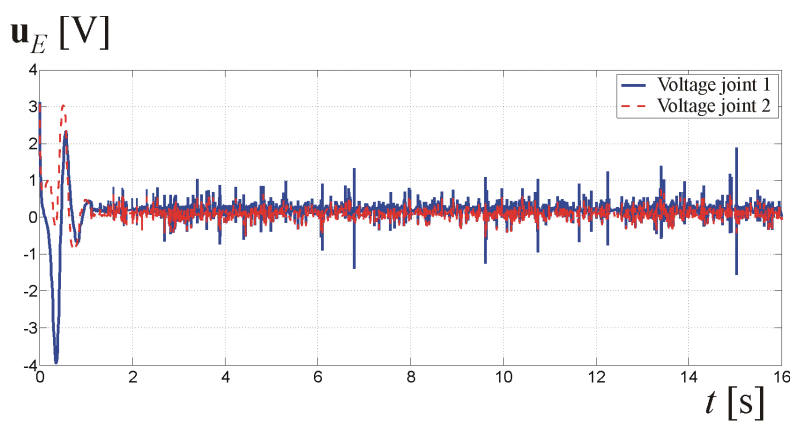

Figure 7: Voltage graph of motors on Pan-Tilt joints 


\section{CONCLUSIONS}

The paper proposes a control method for Pan-Tilt-Camera system driven by DC motors to track a moving object, when there are many uncertainties and noises in system dynamics. An RBF neural network with online learning weights is used to compensate the effects of unknown quantities and noises. The asymptotical stability of the overall system is proven by the Lypunov direct method.

Matlab simulation results show that the proposed algorithm could give satisfied convergence and high precision.

\section{REFERENCES}

[1] W. Yu and M. A. Moreno-Armendariz, "Robust visual servoing of robot manipulators with neuro compensation," Journal of the Franklin Institute, vol. 342, no. 7, pp. 824-838, 2005.

[2] H. Ukida, M. Kawanami, and Y. Terama, "3d object tracking by pan-tilt moving cameras and robot using sparse template matching and particle filter," in SICE Annual Conference (SICE), 2011 Proceedings of. IEEE, 2011, pp. 2004-2009.

[3] T. Wang, G. Liu, and W. Xie, "Visual servoing control of video tracking system for tracking a flying target," in Advanced Intelligent Mechatronics (AIM), 2011 IEEE/ASME International Conference on. IEEE, 2011, pp. 850-855.

[4] C. Zhou and W. Fu, "A study of robot control technology based on stereo vision," in Proceedings of 2011 International Conference on Electronics and Optoelectronics, 2011, pp. 29-31.

[5] H. Wang, Y.-H. Liu, W. Chen, and Z. Wang, "A new approach to dynamic eye-in-hand visual tracking using nonlinear observers," Mechatronics, IEEE/ASME Transactions on, vol. 16, no. 2, pp. 387-394, 2011.

[6] R. Garrido, "Stable neurovisual servoing for robot manipulators," Neural Networks, IEEE Transactions on, vol. 17, no. 4, pp. 953-965, 2006.

[7] P. T. Cat, Modern control methods for industrial robots. The Publishing House of Thai Nguyen University, 2009, in Vietnamese.

Received on October $04-2014$

Revised on August 31 - 2015 